\title{
Spinning and heat-treatment effects on performance and microstructure of maraging steel
}

\author{
HU Zheng-Fei \\ School of Material Science and Engineering, Tongji University, Shanghai 200092, China
}

Keywords: maraging steel; spinning process; plastic deformation; heat-treatment; microstructure. Abstract. The effect of spinning and subsequent heat-treatment on mechanical performance and microstructure evolution has been investigated extensively. Thin-wall tube of $18 \mathrm{Ni} \mathrm{Co}$-free Maraging steel (T250) prepared by forward spinning process emerges obvious radial shrinkage behavior after heat-treatment and the magnitude of the shrinkage induced by solution is almost the same as that by aging. Microstructure investigation shows that the residual stress and stain induced by spinning process and reversed austenite forming during aging might have the similar contribution to the radical shrinkage phenomenon. Solution treatment resulted in a drastically refined grain and recrystallised in microstructure, removing the effect of plastic deformation and improving hardness. Subsequent aging obtained a tempered microstructure, enhancing hardness strikingly for precipitation strengthening.

\section{Introduction}

Maraging steels are well known for their high strength and toughness. In order to investigate their strengthening mechanisms, a variety of experimental techniques were employed, including transmission electron microscopy (TEM), angle X-ray scattering and atom probe field ion microscopy[1,2]. The initial strengthening was caused by the formation of multi-component atomic co-clusters that produced the optimum toughness. The appearance of discrete precipitation of needle-like $\eta$-Ni3Ti particles was associated with a second rise in hardness towards an eventual peak of hardness and strength. Martensite reaction in $\mathrm{Fe}-\mathrm{Ni}$-based alloy induces extremely high solute supersaturations, the microstructures consist of very high density of fine precipitates when aged.

Tube spinning, also known as flow forming, is one of the techniques involving two or three rollers moving along the axial or radial direction and simultaneously deforming the peripheral of axisymmetrical workpiece to the desired shape. Because of its specific benefits, tube spinning has been commonly adopted to reduce the wall thickness of tubular shapes and increase their strength, aiming at aircraft and aerospace products. Music et al.[3] extensively reviewed the spinning process with a thorough survey of academic work on the analysis and application of the mechanics of spinning as well. They reported that the mechanical properties of the product improved considerably. However, the effect of tube spinning on the mechanical properties and microstructure is much less known. In the present study, the deformation percentage of tube wall thickness has reached up to $83.3 \%$, which was applied to no cobalt T250 maraging steel tube preform by forward spinning. The effect of deformation and heat treatments on the mechanical strength and microstructure were investigated. 


\section{Experimental work}

Materials in two states are compared with a view to investigating the shrinkage phenomenon. One material is the preform, a section of hot forged tube, received in solution-annealed state, with dimensions of $200 \mathrm{~mm}$ in diameter and $9 \mathrm{~mm}$ in thickness; another one is a section of thin-wall tube formed out of the preform by forward spinning process with the same diameter but wall thickness reduced to $1.5 \mathrm{~mm}$. In this work, the preform was cold spun from 9.0 to $1.5 \mathrm{~mm}$ by three passes in a flow forming machine with a three roller configuration. The total reduction rate of thickness was $83.3 \%$.

The chemical composition of examined maraging steel is: $18.0 \mathrm{Ni}, 3.0 \mathrm{Mo}, 1.4 \mathrm{Ti}, 0.1 \mathrm{Al}, \mathrm{Fe}$ balance. The examined specimens were cut as slices from the preform and spun tube. Some specimens were given a re-solution treatment at $820^{\circ} \mathrm{C}$ for 1 hour, and part of these re-solution treated specimens were aged at $480^{\circ} \mathrm{C}$ for 4 hours subsequently. Specimens in different states are listed in table 1. The results of a series of heat-treatment practical tests for the spun tube have shown an obvious radial shrinkage. The reduction in radius dimension is about $0.4 \sim 0.65 \mathrm{~mm}$ statistically, $0.2 \sim 0.35 \mathrm{~mm}$ of which comes from solution treatment and the rest $0.2 \sim 0.3 \mathrm{~mm}$ from aging. In contrast, the preform tube did not show any obvious change in radius after heat treatment.

Table 1 Examined specimens in different states

\begin{tabular}{|l|l|}
\hline \multirow{3}{*}{ preform } & P1: $\quad$ as-received \\
\cline { 2 - 2 } & P2: $\quad$ solution treated \\
\cline { 2 - 2 } & P3: $\quad$ solution treated and aged \\
\hline \multirow{3}{*}{ spun } & S1: $\quad$ spun \\
\cline { 2 - 2 } & S2: $\quad$ solution treated \\
\cline { 2 - 2 } & S3: $\quad$ solution treated and aged \\
\hline
\end{tabular}

The Vicker microhardness tests are carried out on polished specimens, and Vicker microhardness was adopted in order to acquire more accurate results for the thin wall spun tube, and the results on average were converted to Rockwell hardness. The hardness test and value conversion are conducted in accordance with relevant ASTM standards.

For light microscopy two etchants were used: Marshall's reagent $(100 \mathrm{ml}$ water, $8 \mathrm{~g}$ oxalic acid, $5 \mathrm{ml}$ $\mathrm{H} 2 \mathrm{SO} 4$, mixed with equal parts of $30 \% \mathrm{H} 2 \mathrm{O} 2$ just prior to etching) and modified Fry's reagent $(50 \mathrm{ml} \mathrm{HCl}, 25 \mathrm{ml} \mathrm{HNO} 3,1 \mathrm{~g} \mathrm{CuCl} 2$ and $150 \mathrm{ml}$ water). The X-ray diffraction was carried out using step scan mode. Radiation $\mathrm{CuK \alpha}(1.54056 \mathrm{~A})$ was used with $40 \mathrm{kV}$ and $100 \mathrm{~mA}$.

Table 2. Vicker microhardness results of the examined samples

\begin{tabular}{|l|l|l|l|l|l|l|}
\hline states & \multicolumn{2}{|l|}{ As-received } & \multicolumn{2}{l|}{ Solution treated } & \multicolumn{2}{l|}{ Solution treated and aged } \\
\hline sample & P 1 & S1 & P2 & S2 & P3 & S3 \\
\hline HV & 327 & 364 & 326 & 327 & 579 & 568 \\
\hline HRC & 33.0 & 37.1 & 32.9 & 33.0 & 54.1 & 53.4 \\
\hline
\end{tabular}

\section{Results and Discussion}

\section{Hardness Test}

Micro-hardness test was conducted on the specimens of different states, so that we can differentiate the effect of spinning and heat treatment on properties, respectively.

Martin [4] reported that maraging steels perform well under cool-forming, with a work-hardening index around 0.02-0.03, and the ranges of hardness in different states, in terms of performance indexes, should not present obvious difference. As listed in Table 2, the hardness of the preform P1 in as-received state is $33 \mathrm{HRC}$, and increases to $37.1 \mathrm{HRC}$ when it was spun as $\mathrm{S} 1$, which primarily 
originates from the spinning process. After the solution treatment, the hardness for both specimens P2 and S2 decrease and become almost the same (33 HRC) as that of initial preform. When these two samples aged at $480^{\circ} \mathrm{C}$ for 4 hours, their hardness increases dramatically (from 30 to about 54 HRC). The hardness for the preform specimen P3 is 54.1 HRC, which is almost equal to that of the spun specimen S3 (53.4 HRC). The hardness difference is very likely due to the microstructure change upon heat treatment, which is discussed in more details below.
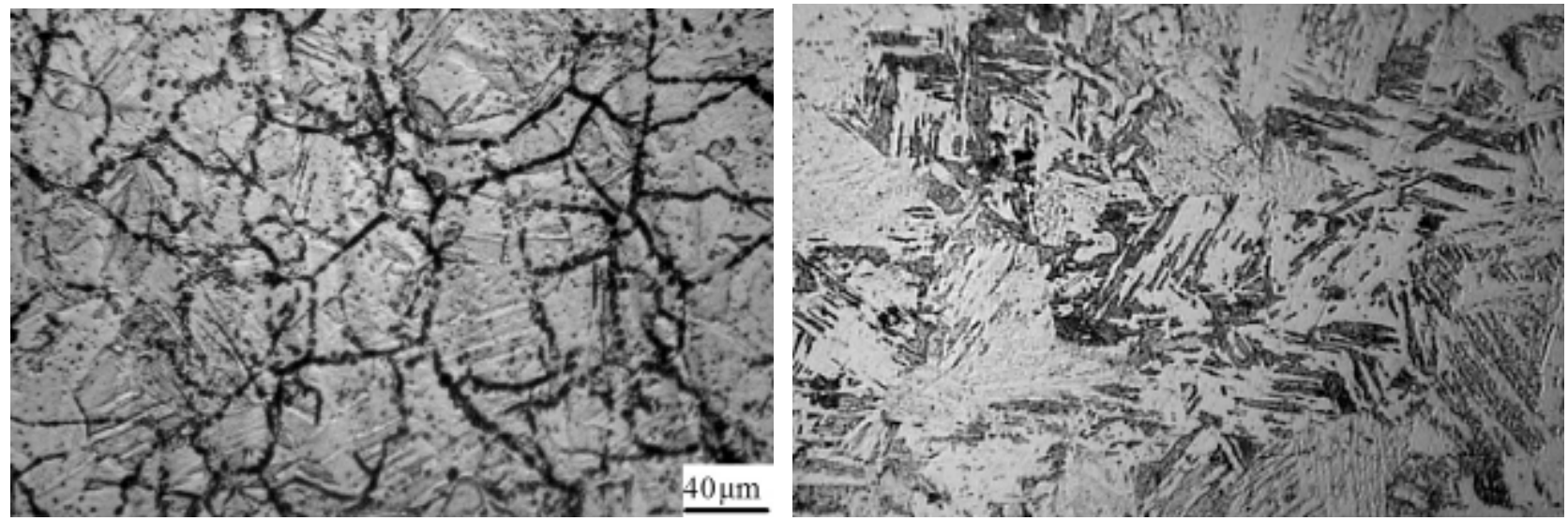

(a)
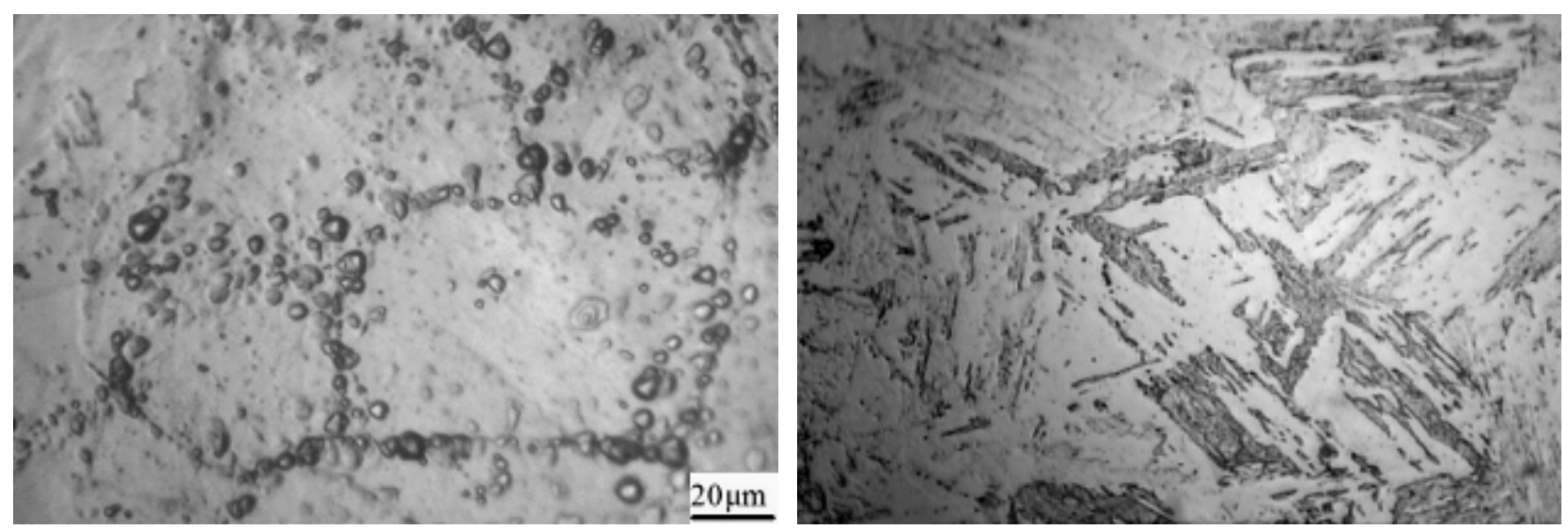

(b)
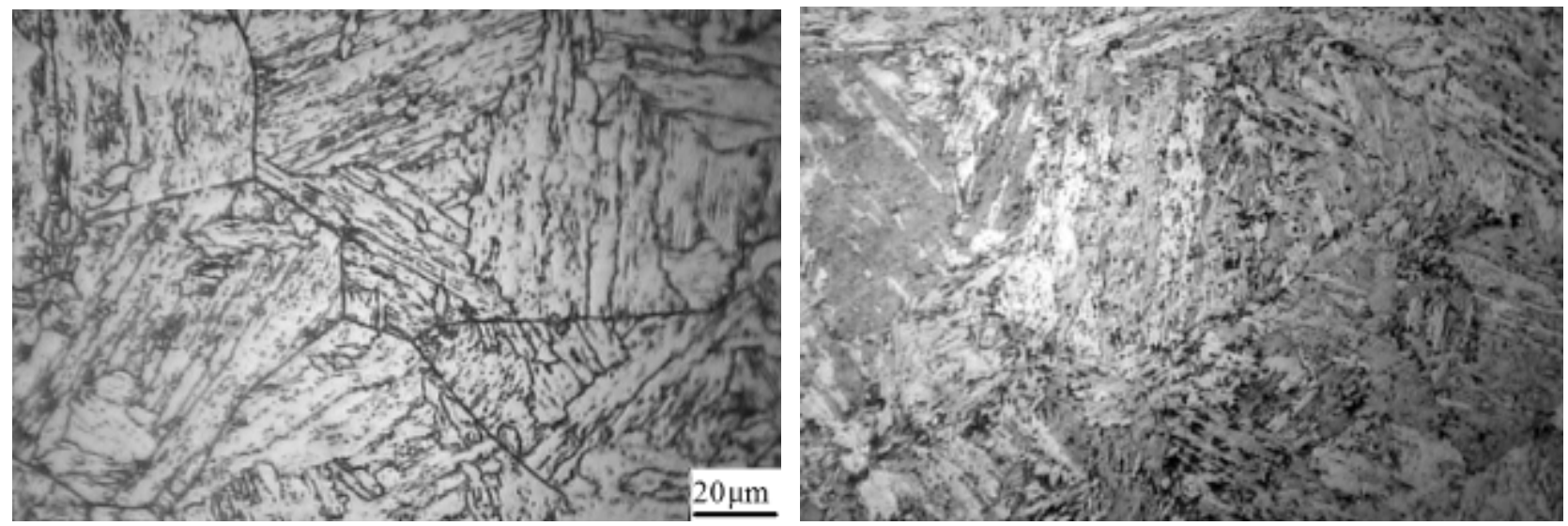

(c)

Fig.1 Optical micrographs of the preform specimens in different states (a) P1, (b) P2, (c) P3. Left: etched using Marshall etchant; Right: modified Fry.

Optical Micrographs 
Fig.1 displays the optical micrographs of the preform in as-received, re-solution treated and subsequently aged states, respectively. All microstructure photographs were taken along the axis of the test specimens. The as-received material was pre-deformed for the emergence of slipping straps (left of Fig.1(a)), and the grains are equilateral, which indicates that the hot extruding process didn't induce obvious deform in microstructure. Furthermore, the presence of wide grain boundaries, as well as the apparent second phases in the matrix, indicates that the as-received alloy was not well normalized.

Fig.1(b) shows the micrographs of the preform after solution treatment at $820^{\circ} \mathrm{C}$. The slipping straps presented in the as-received state disappeared. It also shows that the preform material is a typical lath martensite (right of Fig.1(b)) after solution treatment. That means the second phase in the as-received state didn't dissolve entirely during the solution treatment. After aging, the precipitates distribute dispersively in the matrix, which contributes primarily to the second hardening effect and leads to the drastic increase in hardness (Fig.1(c)).

Micrographs of spun specimen in different states are given in Fig.2 and they were also taken along the axis of the spun specimens. Figs.2(a) and (b) are micrographs of spun specimen in as-received state, which show obvious plastic deforming texture. The isometric morphology in the preform was absent and the deformation during the spinning elongated and refined the grain structure heavily. The elongating orientation can be easily educed as circumferential aspect of tube, which is parallel to the direction in which a mandrel processed. Hardness of the spun specimen increases from 33 to $37.1 \mathrm{HRC}$ as the deformation percentage sets up to $83.3 \%$, which indicates the work hardening effect of the spinning process. In the spinning process, the deformation across the section of a workpiece is not uniform, and sets up large tangential residual stresses in the material, which are tensile according to the report by Gür et al.[5].
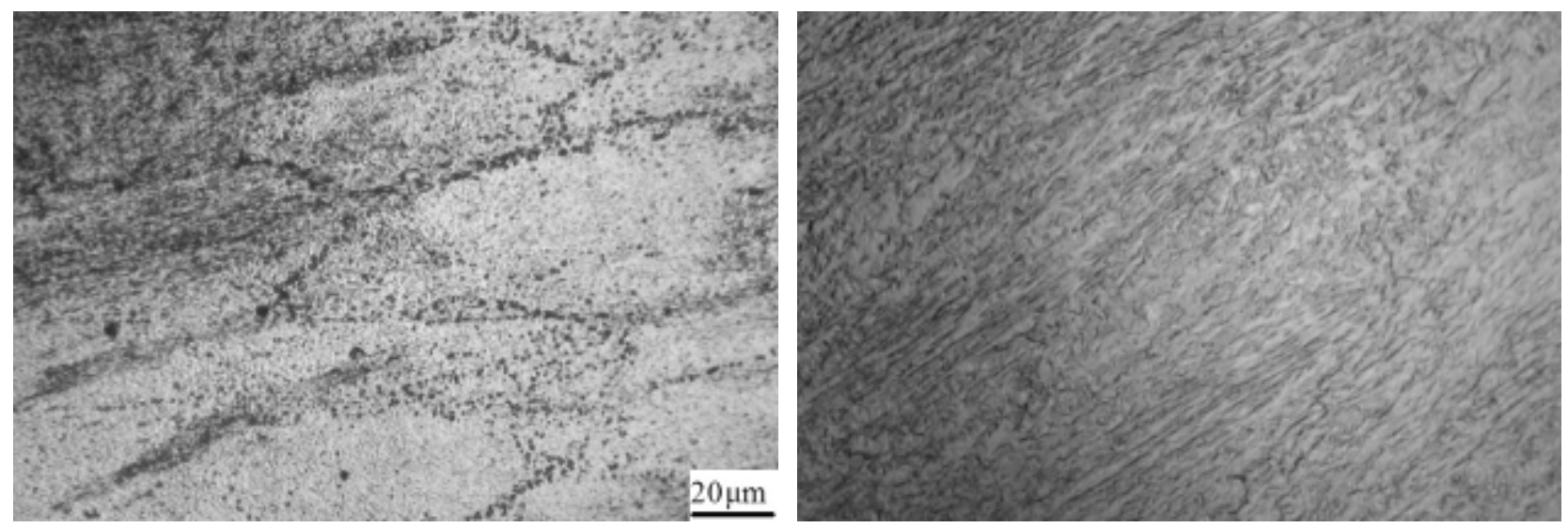

(a)
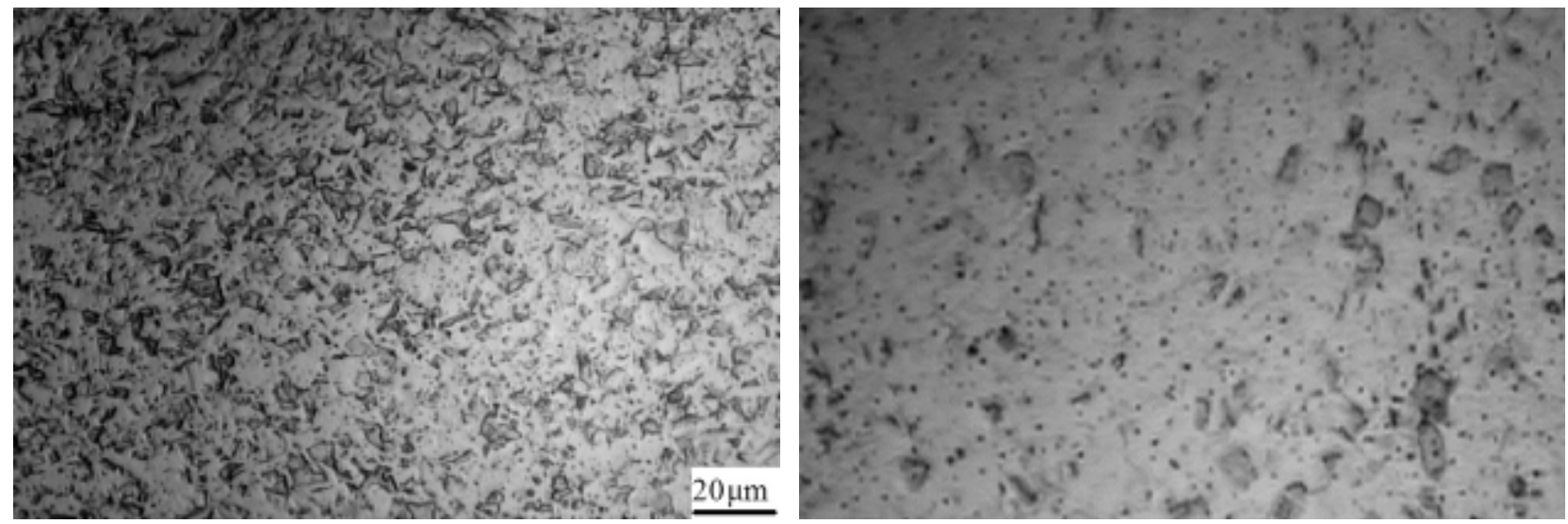
(b)
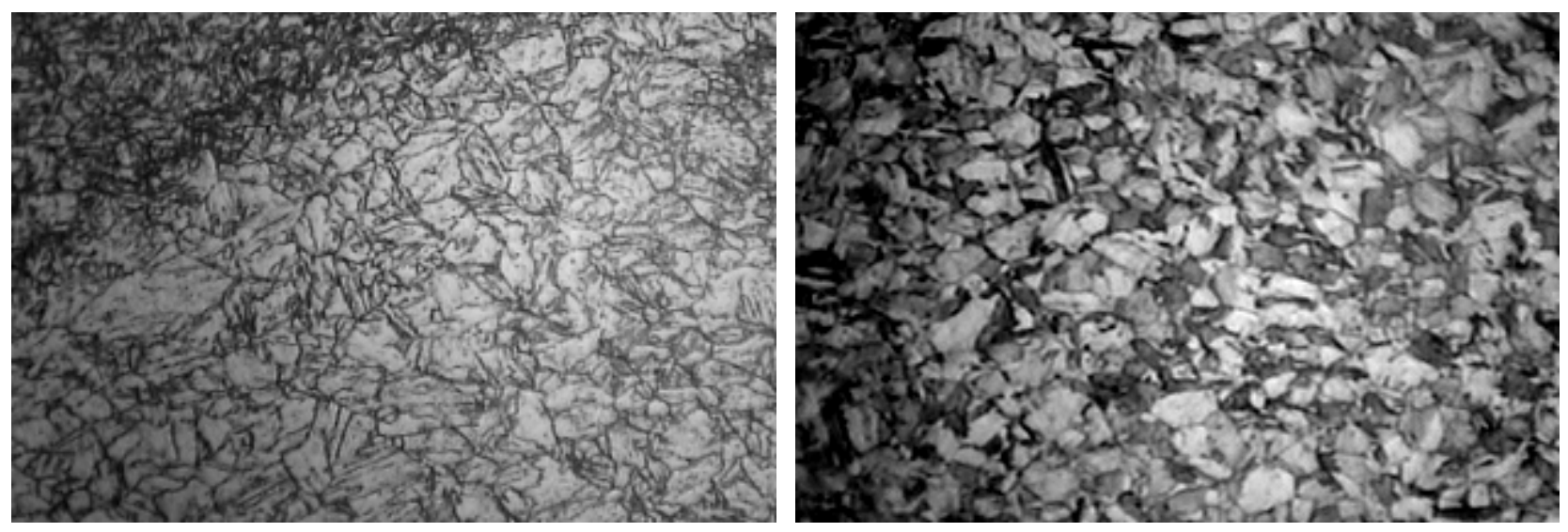

(c)

Fig.2 Optical micrographs of spun specimens in different states (a) S1, (b) S2, (c) S3. Left: etched using Marshall etchant; Right: modified Fry.

Microstructure of the spun specimen after solution treatment is given in Fig.2(b), where the deforming texture disappears and grains are drastically refined to be equilateral. The hardness of the solution treated specimen is $33.0 \mathrm{HRC}$, very close to the initial hardness of the preform. This implies that the microstructure evolution and the residual stress relieving are mainly responsible for the hardness variation in specimen S2. However, the residual stress relieving only causes a slight decrease in the hardness [5], so the variation of hardness should be related to some microstructure morphology. After the solution treatment, the deforming ferrite was consumed completely during the austenite formation and recrystallization, and then the grains were refined severely after quenching. These phenomena cause a recovery in microstructure, reduction in dislocation density, and hardening effect of deformation is counteracted. They are mainly responsible for the decrease in hardness and elimination of tangential residual stress. So the hardness values of specimens S2 and P2 in solution treatment approach each other, which are also close to the initial hardness of perform P1. The hardness does not show distinct dependence on the grain refining in S2, which is consistent with the previous report [6].

After the complete heat treatment (solution treatment + aging), the hardness of specimen S3 increases drastically and is equal to that of $\mathrm{P} 3$, which is due to the second hardening on precipitation. Fig.2(c) displays the featuring lath martensite of S3. It clearly shows that the grains were so smashed and the grain size distribution was not homogenized from one region to another. In the top left corner, grains are much finer than those in the rest regions, which is a characteristic of spinning process for uneven deformation.
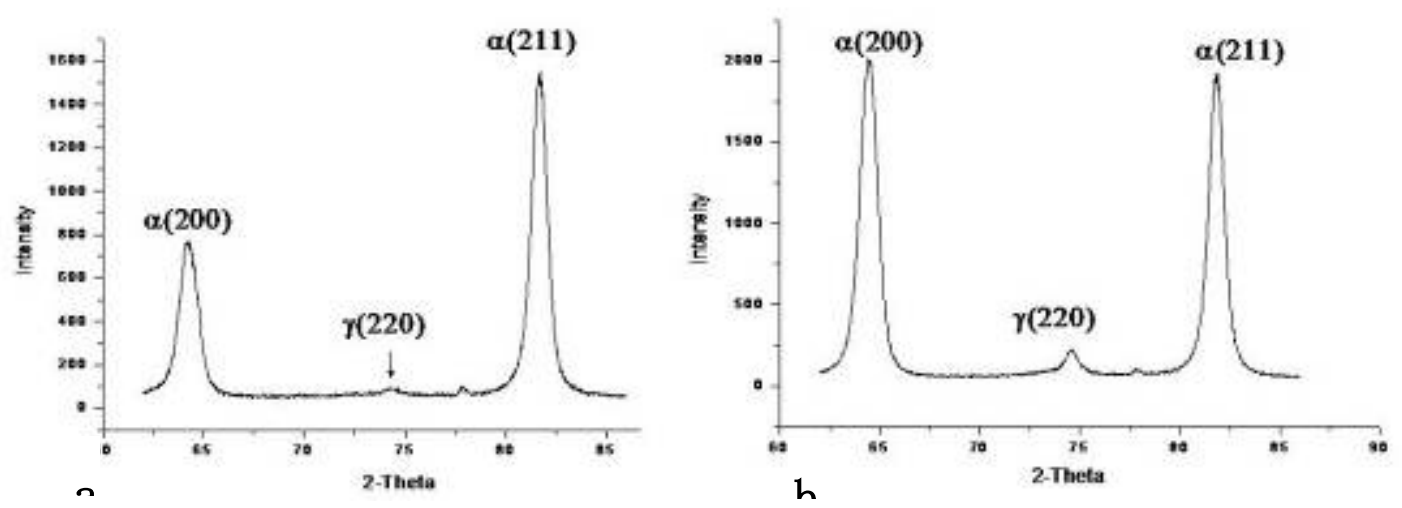

Fig.3 XRD spectra of aging specimens (a) P3, (b) S3. 


\section{XRD Result}

XRD Tests results in Fig.(3) reveal that the examined specimens in different states are $\alpha$-Fe, except that there is a bit of $\gamma$-Fe in the aging specimens of P3 and S3. The peaks of (200) $\alpha,(211) \alpha$ and (220) $\gamma$ can be easily seen from diffraction diagrams. Without considering precipitates, we can easily obtain the relative contents of $\gamma$-Fe in an alloy with only two phases by direct comparison method [7]. From the data of relative diffracted intensities, the austenite volume fraction is about $3 \%$ in $\mathrm{P} 3$, and $7 \%$ in S3. This result clearly shows that the samples P3 and S3 consist of different amounts of austenite even under the same heat treatment routines, which is obviously related to their different processing.
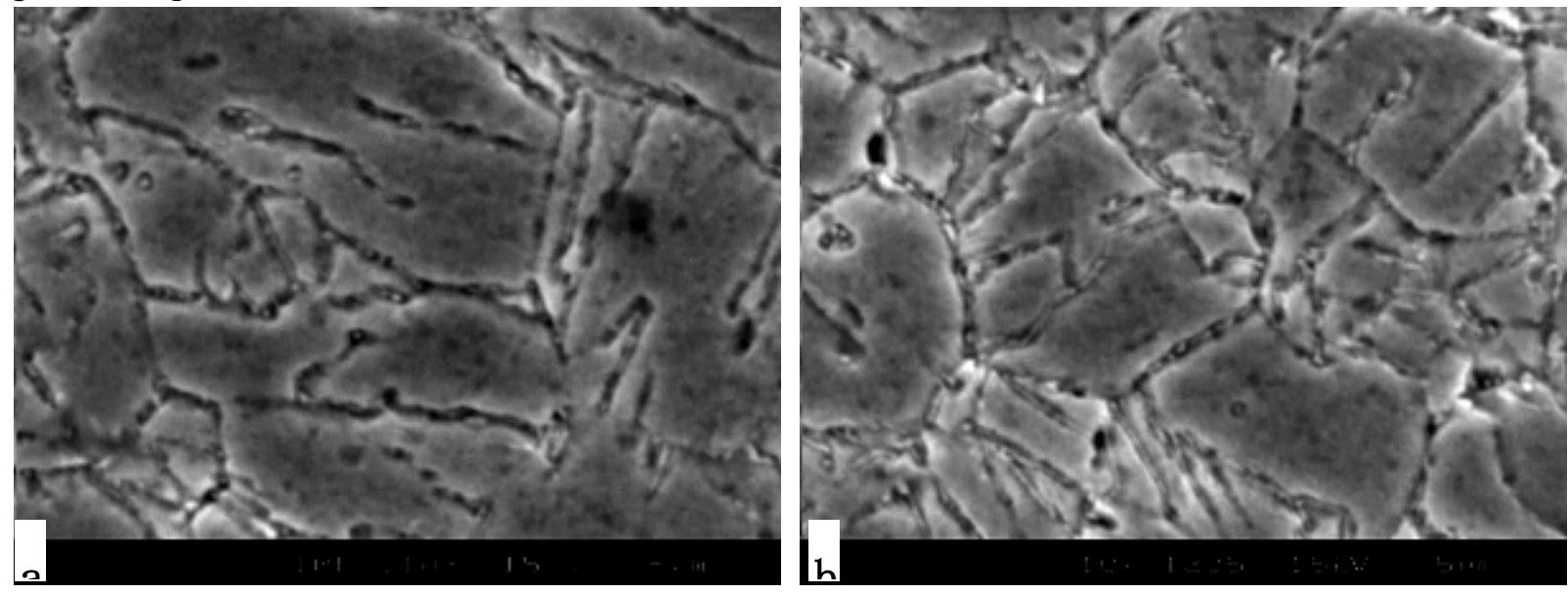

Fig.4 SEM micrograph of specimens (a) P3 and (b) S3, etched using Marshall etchant.

Shamantha et al. [8] reported that SEM micrographs could reveal the austenite distribution in maraging steel that is in the form of plate along grain and lath boundaries. Fig. 4 shows clear grain boundaries of specimens P3 and S3, with a significant reverted austenite distributing in the boundaries. Clearly, much more austenite exists in sample S3 for its larger boundary area. From the above observations, it is quite natural to interpret the visible radial shrinkage to the effects of working process and subsequent heat treatment. Spinning process, a kind of local plastic distortion, has a high stress on the working object with a unit stress of 2500-3500 N/mm2. Under this circumstance, high stress and residual strain gather in the matrix. It explains that the hardness of specimen from spun tube is higher than that of initial preform. Heat treatment relieves the residual stress and strain completely, so the microstructure is arranged in a more orderly way, which may partly contribute to the radial-shrinkage (Fig.2a to Fig.2c).

In addition to the effects of deforming defects abatement and precipitating reaction during heat treatment, one distinguished feature is that final specimen S3 contains more austenite, most of which is reversed austenite formed during aging and the rest is retained austenite from solution stage. According to the report by Moyer et al. [9], the volume of steel can reduce by $3 \%$ after the $\alpha-\gamma$ transformation with same atoms. So the microstructure evolution also influences the shrinkage behavior to a certain degree. The fact that the volume fraction of austenite in spun specimen S3 is more than that in the preform P3 may be related to the grain refining detail in S3, because the reversed austenite formed in grain and lath boundaries, and the grain refined specimen S3 has several times boundary area than that in P3. Statistically, the radial shrinkage resulting from solution treatment is identical with that from aging. Therefore, the radial shrinkage could originate from processing residual press, strain releasing in solution heat-treatment and reversed austenite formed during aging.

Overall, the specimens P2 and S2 after solution treatment, and the specimens P3 and S3 after solution treatment and aging have displayed almost the same hardness. This observation implies 
that the mechanical properties of examined maraging steel are not closely related to grain size, which is consistent with the previous reports by Sinha et al. [10]. It is believed that the lath widths are similar even though the grain sizes are different and the strengthening effect of lath is prominent for maraging steels in solution state. Our observation shows the microstructures in the preform and spun samples are different after complete heat-treatment. The former possesses large grains with tiny austenite, and the latter displays much smaller grains with more austenite. So the quality of reversed austenite is another source that induces the same hardness in maraging steels with different grain size. On the other hand, ageing process can induce hardness increase dramatically, which means that the precipitates have more significant strengthening effect than grain refining effect. The nanometer-size precipitates were identified as $\eta-\mathrm{Ni} 3 \mathrm{Ti}$, which dispersed in certain orientation in the matrix homogenously $[2,11]$.Though the exact function of precipitates in the radial-shrinkage is not clear, they may have little to do with this phenomenon for their minimal volume fraction.

\section{Conclusion}

(1) Maraging steel tubes formed by spinning arouse significant radial shrinkage during heat-treatment, and the solution treatment induced shrinkage is equal to that by aging. which is mainly related to deforming process and microstructure evolution. During solutionizing, the shrinkage is most because of residual press and strain released which created by heavy deformation, while aging, the shrinkage is primarily caused by the formation of reversed austenite.

(2) The microstructures in the preform and spun tubes are profoundly different upon complete heat-treatment. The former has large grains with tiny austenite, and the latter possesses superfine grains with relatively more austenite. Both hardness is not obvious difference, which implies that the mechanical properties of maraging steel are not closely related to grain size.

\section{References}

[1]E. V. Pereloma, A. Shekhter, M. K. Miller and S. P. Ringer:Acta Mater. Vol.52 (2004), p.5589.

[2]V. K. Vasudevan, S. J. Kim and C. M. Wayman: Metal. Trans. Vol. A21 (1990), p.2655.

[3]O. Music, J. M. Allwood and, K. Kawai: J. Mater .Process Technol. Vol.210 (2010), p.3.

[4]J. W. Martin: Micromechanisms in particle hardened alloys (Cambridge University Press, Cambridge 1980).

[5]C. H. Gür and E. B. Arda :Mater. Sci. \& Tech. Vol.19 (2003),p.1590.

[6] H. J. Rack: Mater. Sci. \& Eng. Vol.34 (1978), p.263.

[7]J. M. Pardal, S. S. M. Tavares, M. P. C. Fonseca, H. F. G. Abreu and J. J. M. Silva: J. Maters. Sci. Vol.52 (2006), p2301.

[8]C. R. SHAMANTHA, R. Narayanan, K. J. L. Iyer, V. M. Radhakrishnan, S. K. Seshadri, S. Sunderrajan and S. Sundaresan: Mater. Sci. \& Eng. Vol.A287 (2000), P. 43.

[9]J. M. Moyer and G. S. Ansell: Metall. Trans. Vol. A6 (1975), P.1785.

[10]P. P .Sinha, K. T. Tharian, K. Sreekumar, K. V. Nagarajan and D. S. Sarma: Mater. Sci. Tech.Vol.14 (1998),P.1.

[11]D. W. Vanderwalker: Metal. Trans. Vol.A18 (1987), p.1194. 